\title{
Bloodletting Therapy
}

Avicenna (translated by Molla Fathollah ibn Fakhr-e-din Shirazi)

\section{Source}

Avicenna (translated by Molla Fathollah ibn Fakhr-e-din Shirazi). (1928). The Canon of

Medicine JISHIM, vol. 8

WCT is a procedure for bloodletting from the capillary networks after sucking and scarification steps. In this procedure, a cup is mostly attached to the surface of the skin by using a negative pressure or suction- like force. A few minutes later, the uplifted cup is removed, and superficial incisions are made using a scalpel. The cup is then replaced, and the procedure is repeated three to five times until some blood and interstitial fluid are drained.

According to Avicenna (Abu Ali Sina), wet cupping (Hijamat, in his own term) acts through two mechanisms: first, blood purification or clearance, especially for the skin and its adjacent organs, and second, thin blood drainage. 